\title{
Effective field theory of the disordered Weyl semimetal
}

\author{
Alexander Altland and Dmitry Bagrets ${ }^{1}$ \\ ${ }^{1}$ Institut für Theoretische Physik, Universität zu Köln, Zülpicher Str. 77, D-50937 Köln, Germany
}

(Dated: January 27, 2015)

\begin{abstract}
In disordered Weyl semimetals, mechanisms of topological origin lead to the protection against Anderson localization, and at the same time to different types of transverse electromagnetic response - the anomalous Hall, and chiral magnetic effect. We here apply field theory methods to discuss the manifestation of these phenomena at length scales which are beyond the scope of diagrammatic perturbation theory. Specifically we show how an interplay of symmetry breaking and the chiral anomaly leads to a field theory containing two types of topological terms. Generating the unconventional response coefficients of the system, these terms remain largely unaffected by disorder, i.e. information on the chirality of the system remains visible even at large length scales.
\end{abstract}

PACS numbers: 75.47.-m, 03.65.Vf, 73.43.-f

Weyl semimetals are paradigmatic examples of gapless topological condensed matter systems. A Weyl semimetal comprises an even number of linearly dispersive band touching points embedded in a threedimensional Brillouin zone. The presence of these hotspots implies a response to perturbations that is intermediate between that of metals and insulators (see Ref. [1] for review). This 'semi-metallicness' also signifies in the physics of the disordered system [2 [5]: on the one hand, the vanishing of the nodal density of states weakens disorder scattering cross sections; on the other hand, sufficiently strong disorder will generate a finite band center density of states to eventually overpower the above effect. It has been shown [4 that the above competition manifests itself in the presence of a critical disorder strength below/above the system flows towards a clean fixed point/a regime of strong impurity scattering. It is the purpose of the present paper to derive and discuss the effective theory describing the latter phase at length scales exceeding the system's scattering mean free path.

At large length scales, impurity scattering will render the motion of individual excitations diffusive which suggests that the system will end up in the universality class of the $3 \mathrm{~d}$ Anderson metal (i.e. above the phase transition point separating a $3 \mathrm{~d}$ metal from an insulator.) This expectation is, in fact, a certainty given that a single Weyl node may be interpreted as an effective surface theory of a bulk 4d topological insulator; finite conduction is protected by topology. At the same time, topology implies a number of differences distinguishing the Weyl system from a generic metal: First, an individual Weyl node breaks parity symmetry, and it is known (e.g. from the example of the $d$-wave superconducting quasiparticle system [6]) that the breaking of discrete symmetries is generally remembered, even in the presence of strong disorder. Indeed, we will find that the low energy theory of individual nodes system contains a parity breaking non-abelian Chern-Simons (CS) term, which describes the survival of the so-called chiral magnetic effect (CME) [7, 8] in the disordered environment. Second, it has been shown that a system comprising two Weyl nodes separated in momentum space shows an anomalous Hall effect (AHE) 9. Within the field theoretic framework below, this effect will derive from a $3 \mathrm{~d}$ extension of a two-dimensional topological $\theta$-term, familiar from the theory of the quantum Hall effect.

Field theory - In the following, we will derive an effective feld theory describing these structures. Our starting point is the bi-nodal Hamiltonian (cf. Fig. 1)

$$
\hat{H}=v \hat{k} \sigma_{3}^{\mathrm{n}}+(v \not b+\mu)+V(\mathbf{x})
$$

where $\hat{k} \equiv \mathbf{k} \cdot \boldsymbol{\sigma}, \boldsymbol{\sigma}$ is a vector of Pauli matrices, $\hat{\mathbf{k}}$ the vector momentum operator, and $v$ a characteristic velocity. The Pauli matrix $\sigma_{3}^{\mathrm{n}}$ acts in a two-component space discriminating between two nodes split by a vector $2 \mathbf{b} \equiv 2 b \mathbf{e}_{3}$ in momentum space and an increment $2 \mu$ in energy. The model is coupled to disorder by a Gaussian distributed potential $V(\mathbf{x})$ with variance $\gamma_{0}$. We discriminate between disorder correlated over length scales $\gtrsim b^{-1}$, which is soft in the sense that the two Weyl nodes are not coupled by impurity scattering, and the opposite case of short range correlated disorder mixing the nodes. Implicit to the model is a high momentum cutoff $|\mathbf{k}|<\Lambda$ limiting the range of linearizability of an underlying lattice model.

To access the transport properties of the system at energies $\epsilon$, we introduce a supersymmetric generating functional $Z[a]=\int D(\bar{\psi}, \psi) \exp (-S[\bar{\psi}, \psi])$, with action $S[\bar{\psi}, \psi, a]=-i \int d^{3} x \bar{\psi}\left(\epsilon+i \delta \tau_{3}-\hat{H}\right) \psi$. Here $\psi=$ $\left\{\psi_{s, i, n}^{\alpha}(\mathbf{x})\right\}$ is a sixteen-component field of integration variables where, $n=1,2$ labels the two nodes, $i=1,2$ the components of a Weyl spinor, $s= \pm$ distinguishes between advanced and retarded (ar) Green functions, i.e. $\left(\tau_{3}\right)_{s s^{\prime}}=s \delta_{s s^{\prime}}$ such that integration over $\psi_{s= \pm}$ generates matrix elements of $\hat{G}^{ \pm}=(\epsilon \pm i \delta-\hat{H})^{-1}$, and $\alpha=1,2$ defines a two-dimensional space (bf-space) of complex commuting/Grassmann variables $\psi^{1 / 2}$. In the commuting sector, $\bar{\psi}^{1}=\psi^{1 \dagger}$, while the commuting variables $\bar{\psi}^{2}, \psi^{2}$ are independent Grassmann variables. From 
$Z$, transport observables may be computed by introducing suitably defined source fields $a$, which are presently suppressed to keep the notation simple.

To explore the influence of disorder on the system, we integrate over $V$ to generate the quartic contribution $\frac{\gamma}{2} \int d x(\bar{\psi} \psi)^{2}$. The fate of this nonlinearity under changes of the cutoff $\Lambda$ has been studied [5, 10] by evaluating the results in one- and two-loop renormalized perturbation theory in $2+\epsilon$ dimensions [11] at $\epsilon=1$. It has been found that for bare amplitudes larger than a critical value $\gamma^{*}=\pi^{2} v^{2} / \Lambda$ the effective disorder strength increases under renormalization. To describe the perturbatively inaccessible regime beyond the scattering mean free path, $\Lambda^{-1} \equiv l \sim \gamma / v^{2}$, we decouple the nonlinearity by a supermatrix field $B$, and integrate over the then quadratic fermions [12]. As a result, we obtain the effective action $S[B]=\frac{1}{2 \gamma} \int d^{3} x \operatorname{str} B^{2}-\operatorname{str} \ln (\hat{G}[B])$, where $\hat{G}[B]=\left(\epsilon+i \delta \tau_{3}-\hat{H}_{0}-B\right)^{-1}, \hat{H}_{0}$ is the clean Hamiltonian and str is the supertrace [12]. The difference between the cases of hard and soft disorder, resp., is that in the former/latter case the two nodes couple to the same $\left(B=B \otimes \mathbb{I}^{\mathrm{n}}\right) /$ independent $\left(B=\operatorname{bdiag}\left(B_{1}, B_{2}\right)^{\mathrm{n}}\right)$ matrix fields. For definiteness, we first consider the soft case, for which the two nodes can be discussed separately; the effect of impurity mixing can be described by a locking $B_{1}=B_{2}$ at any later stage. Focusing on node $n=1$ and writing $B=B_{1}$ for notational simplicity, we proceed by subjecting the action to a mean field analysis. A variation of the action yields the equation $\bar{B} \stackrel{!}{=} \gamma \operatorname{tr} \hat{G}(\mathbf{x}, \mathbf{x} ;[\bar{B}])$, which is solved [11] by the diagonal ansatz, $\bar{B}=-i \kappa \tau_{3}$ describing the 'spontaneous symmetry breaking' of the infinitesimal $\delta$ a finite impurity self energy $\kappa=\kappa(\epsilon)$. Specifically, at $\epsilon=0$ (semimetal) one finds $\kappa=(2 / \pi) v \Lambda\left(1-\gamma^{*} / \gamma\right)$ [13, while far away from Weyl node (metal) $\kappa=\gamma \pi \nu$, where $\nu=\epsilon^{2} / 2 \pi^{2} v^{3}$ is the clean density of states.

Turning to the analysis of fluctuations around the mean field, we focus on configurations $B \equiv i \kappa Q \equiv$ $i \kappa T \tau_{3} T^{-1}$, whose action vanishes in the limit of slowly varying fluctuation matrices $T(\mathbf{x}) \rightarrow$ const. Here, $T \in$ $G / H$ takes values in a Goldstone mode coset space where $G=\operatorname{GL}(2 \mid 2)$ is the group of $4 \times 4$ invertible supermatrices and $H=\mathrm{GL}(2 \mid 2)$ the subgroup of matrices $k$ commutative with the mean field, $\left[k, \tau_{3}\right]=0$. Since $\operatorname{str}\left(Q^{2}\right)=0$, the action we now need to consider reads $S_{0}[Q] \equiv \operatorname{str} \ln (\epsilon-v / k+i \kappa Q)$. For later reference, we note that the action has two continuous symmetries: a global symmetry $Q \rightarrow T_{0} Q T_{0}^{-1}$, where $T_{0} \in G$ is constant, and a local gauge symmetry $T \rightarrow T k, k=k(\mathbf{x}) \in H$.

Effective field theory - Our goal is to expand the action in gradients $T^{-1} \partial_{i} T \equiv A_{i}$. One might be tempted to start this program with a similarity transformation, $S_{0}[Q] \stackrel{?}{=} \operatorname{str} \ln \left(T^{-1}(\epsilon-v \not k+i \kappa Q) T\right)=\operatorname{str} \ln (\epsilon-v \not k+i v \not A+$ $\left.i \kappa \tau_{3}\right) \equiv S[A]$, which may then be expanded in powers of the 'non-ablian gauge field' $A$. However, due to the noto-

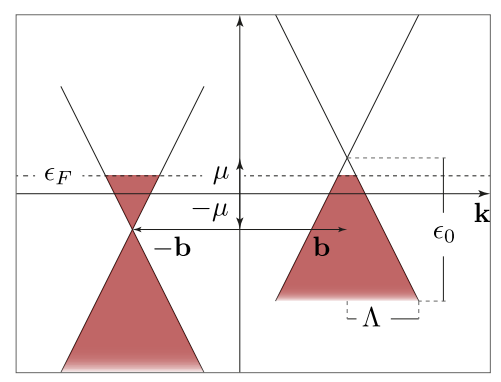

Figure 1. (Color online) Schematic of two Weyl nodes split in energy and momentum by $2 \mu$ and $2 \mathbf{b}$, resp.

rious anomaly, this operation is invalid, the action needs to be regularized first. Following a strategy previously applied to the $2 \mathrm{~d} d$-wave superconductor, we regularize by subtraction of a term $S[Q] \equiv S_{0}[Q]-S_{\eta}[Q]$, where $S_{\eta}$ differs from $S_{0}$ by a replacement $\kappa \rightarrow \eta \searrow 0$ and setting $\epsilon=0$. In the limit $\eta \rightarrow 0$ the $Q$-dependence of $S_{\eta}[Q]$ drops out so that $S_{\eta \rightarrow 0}[Q]=0$. On the other hand, for large momenta $v|k| \gg q$ and fixed $\eta$, the two action contributions cancel against each other, i.e. $S[Q]$ is UV regularized (in a gauge-invariant way.) The similarity transformation may now safely be applied to both $S_{0, \eta}$, so that we obtain an effective action $S[Q]=\left(S_{0}[A]-S_{\eta}[A]\right)_{\text {reg }}$, where the subscript 'reg' means that only UV finite contributions to the subsequent expansion in $A$ are to be kept. Notice that in the language of the $A$-fields, the local gauge invariance is no longer manifest. Rather, $A_{i} \rightarrow k^{-1}\left(A+k \partial_{i} k^{-1}\right) k$ transforms as a non abelian gauge field, and invariance of the action becomes a nontrivial consistency condition.

In the expansion of the action, we keep terms of order two $\left(\mathcal{O}\left(\partial A, A^{2}\right)\right)$ and three $\left(\mathcal{O}\left(A^{3}, \partial A\right)\right)$ derivatives. To second order we obtain the result

$$
\begin{aligned}
S_{\mathrm{d}}[A] & =-\frac{\sigma_{x x}^{1}}{8} \sum_{i} \int d^{3} x \operatorname{str}\left(\left[A_{i}, \tau_{3}\right]^{2}\right), \\
S_{\text {top }}[A] & =\frac{\sigma_{x y}^{1}}{2} \epsilon^{3 i j} \int d^{3} x \operatorname{str}\left(\tau_{3} \partial_{i} A_{j}\right),
\end{aligned}
$$

where the longitudinal and Hall conductivity of node 1, $\sigma_{x x}^{1}$ and $\sigma_{x y}^{1}$ are determined by the microscopic model parameters as discussed below. We note that the action (2) affords the manifestly gauge invariant reformulation $S_{\mathrm{d}}[Q]=-\frac{\sigma_{x x}}{8} \int d^{3} x \operatorname{str}\left(\partial Q^{2}\right)$ and $S_{\mathrm{top}}[Q]=$ $\frac{\sigma_{x y}}{8} \epsilon^{3 i j} \int d x \operatorname{str}\left(Q \partial_{i} Q \partial_{j} Q\right)$. The first of these contributions has been constructed in Ref. [11] on phenomenological grounds within a non-regularized framework. (In our current approach regularization plays a vital role; the 'cross term' $A \tau_{3} A \tau_{3}$ of the commutator $\left[A, \tau_{3}\right]^{2}$ is weighed with an UV divergent 'fermion bubble'. Only after regularization it combines with the finite coefficient of $A^{2}$ to a gauge invariant commutator.) Before discussing the physics of these expressions, we complete the derivation of the action and consider terms of cubic order in $A$. 
The terms of $\mathcal{O}\left(A^{3}\right)$ are the 'triangle graphs' pervasive in the theory of $(2+1)$ or $(3+0)$ dimensional relativistic gauge theories. On general grounds [14] we expect the appearance of a Chern-Simons action at this order. A straightforward if lengthy calculation indeed yields the result

$$
\begin{aligned}
S_{\mathrm{CS}}[A] & =S_{\mathrm{CS}}^{I}[A]+S_{\mathrm{CS}}^{I I}[A] \\
S_{\mathrm{CS}}^{I}[A] & =\frac{i \epsilon^{i j k}}{8 \pi} \sum_{s= \pm} s \int d^{3} x \operatorname{str}\left(A_{i} P^{s} \partial_{j} A_{k} P^{s}\right) \\
S_{\mathrm{CS}}^{I I}[A] & =\frac{i \epsilon^{i j k}}{12 \pi} \sum_{s= \pm} s \int d^{3} x \operatorname{str}\left(A_{i} P^{s} A_{j} P^{s} A_{k} P^{s}\right)
\end{aligned}
$$

where $P^{ \pm}$is a projector on advanced/retarded indices. Apart from the presence of these projector matrices, this has the characteristic structure of a non-abelian CS term. (However, (3) does not define a 'real' CS action, inasmuch as $A$ does not describe a field-gauge coupling, but represents the nonlinear $\sigma$-model field itself; the situation is conceptually similar to that considered in Refs. [15, 16].)

The CS action does not afford a representation in terms of $Q$-fields, which reflects the lack of complete gauge invariance of this action piece [14]: it is straightforward to verify that under a gauge transformation by $k \equiv \operatorname{bdiag}\left(k_{+}, k_{-}\right)^{\text {ar }} \in H$, and for a fictitious system without boundaries, the CS action transforms as $S_{\mathrm{CS}}[A] \rightarrow S_{\mathrm{CS}}[A]+S_{\mathrm{top}}[k]$, where $S_{\mathrm{top}}[k]=$ $\frac{-i}{24 \pi} \sum_{s= \pm} s \int d^{3} x \operatorname{str}\left(k_{s}^{-1} \partial k_{s}\right)^{\wedge 3}$. The integral yields a quantized value, viz. $24 \pi^{2} \times n_{s}$, where $n_{s}$ is the is winding number of a configuration $k_{s}^{\mathrm{ff}} \in \mathrm{SU}(2)$ in three dimensional space. (The non-compact bb sector of the supermatrices $k$ is topologically empty.) For 'large' gauge transformations with non-vanishing winding numbers, the CS action changes by a factor $i \pi\left(n_{+}+n_{-}\right)$. The origin of this phenomenon was explained in the classic reference [14, where it was shown that these factors get canceled by a gauge anomaly of the regulator action $S_{\eta} \rightarrow S_{\eta}+i \pi\left(n_{+}+n_{-}\right)$, where the appearance of the extra terms is caused by zero-crossings of the regularizing Dirac operator under a large gauge transformation. Overall the action is gauge invariant.

Discussion - The action $S_{d}+S_{\text {top }}$ of the diffusion modes $A$ appeared for the first time in connection with the multi-layer quantum Hall effect [17, a system conceptually similar to the present one if the 3-direction of Weyl node splitting is interpreted as the stacking direction of a layered system of $2 d$ class A topological insulator layers [9]. The action $S_{\mathrm{d}}$ controls the fluctuations of diffusion modes in terms of the dimensionless coupling constant $g_{x x} \equiv \sigma_{x x}^{1} \Lambda^{-1}$ Within the framework of our gradient expansion we find $\sigma_{x x}=\left(\epsilon^{2}+3 \kappa^{2}\right) / 6 \pi \kappa v$, which simplifies to $\sigma_{x x}^{1}=\kappa / 2 \pi v$ at the Weyl node and asymptotes to the Drude conductivity $\sigma_{x x}^{1}=v^{2} / 3 \gamma$ at higher energies $\epsilon \gg \kappa$. At the nodes, $\epsilon=0$, and at bare length scales $\Lambda \sim l^{-1}$ characteristic for the ballistic/diffusive crossover, the conductance $g_{x x}$ takes values of $\mathcal{O}(1)$, close to but larger [18] than the critical value $g^{*}$ marking the $3 \mathrm{~d}$ Anderson transition.

The bare coefficient $\sigma_{x y}^{1}$ multiplying the action $S_{\text {top }}$ is the contribution of node 1 to the Hall conductivity of the system at crossover length scales to the diffusive regime. As in the derivation of the field theory of the quantum Hall effect [19] we obtain $\sigma_{x y}$ as a thermodynamic coefficient $\sigma_{x y}=V^{-1} \partial_{B} N$, where $V \equiv L_{z} L^{2}$ is the volume of the sample, and the derivative probes the sensitivity of the number of states $N=$ $-\frac{1}{\pi} \int_{-\infty}^{0} d \epsilon \operatorname{Im} \operatorname{tr}\left(G^{+}(\epsilon)\right)$ below zero energy to the presence of an external magnetic field in 3-direction. Within the framework of the gradient expansion, $G^{+}$is the retarded Weyl node Green function coupled to disorder in the mean field/self consistent Born approximation. Following the strategy of Ref. [9], we evaluate this expression by interpreting the quantized values of the 3momentum, $m_{n} \equiv v\left(b+k_{3, n}\right), k_{3}=2 \pi n L_{z}^{-1}$ as masses entering effective $2 d$ layered Dirac Hamiltonians governing the planes perpendicular to the 3-direction. Within this interpretation, $\sigma_{x y}=\left(2 \pi / L_{z}\right) \sum_{n} \sigma_{x y, n}$ is obtained by summing over the contribution of the layers, where $\sigma_{x y, n}=C_{n} / 2 \pi$ is given by the disorder averaged Chern number, $C_{n}=(1 / 2 \pi) \sum_{\sigma= \pm} \arctan \left[\left(m_{n}+\sigma \epsilon\right) / \kappa\right]+1 / 2$, of the $n$th layer. Since only changes of $C_{n}$ at zero crossings of the effective masses can be unambiguously determined from the linearized theory, we fix the absolute value of the sum by the condition $\sigma_{x y}=0$ at $b=0$, which follows from matching to the band structure of the bi-nodal Weyl system. In the limit $L_{z} b \gg 1$, this gives $\sigma_{x y}^{1 / 2}=b / 2 \pi$ for both nodes, irrespective of the energy $\epsilon$ or the disorder strength $\kappa$. Both for soft and hard disorder, the two contributions to the Hall conductivity add, and we obtain $\sigma_{x y}=b / \pi$, a result known as the anomalous Hall effect (AHE).

The CS contribution to the action accounts for the thermodynamic response of the system to imbalances $(\mathbf{b}, \mu)$ between the nodes. That this term probes equilibrium properties follows from the presence of the projector matrices $P^{s}$ which prevent coupling between the retarded and advanced sector of the theory (a necessary ingredient to any type of dynamic response.) A finite equilibrium response is obtained if we couple the system to an external field $a=\left\{a_{i}\right\}$, where $a_{i}=\frac{B}{2} \epsilon_{3 i j} x^{j}$, $i=1,2$ represents an external magnetic field $B \mathbf{e}_{3}$, and $a_{3}=a(x) \sigma_{3}^{\mathrm{bf}} \otimes \tau_{3}$ is a source field. The latter is defined in such a way that differentiation of the partition function, $\frac{i}{4 \pi} \delta_{a(\mathbf{x})} Z[a]=-\frac{1}{\pi} \operatorname{Im}\left\langle\operatorname{tr}\left[G^{+}(\mathbf{x}, \mathbf{x}) \sigma^{3}\right]\right\rangle=$ $\left\langle j_{3}(\mathbf{x}) \delta(\epsilon-\hat{H})\right\rangle \equiv j_{3, \epsilon}$ yields the contribution of states at energy $\epsilon$ to the equilibrium value of the 3 -current density of node 1 . We compute this expression by adding the external field to the internal one, $A \rightarrow A+a$, and substituting this configuration into the CS action. In the simplest approximation $A=0$ (for the above 'equilibrium' 
choice of source terms fluctuation corrections around the $A=0$ vanish by supersymmetry anyway), we then obtain $S_{\mathrm{CS}}[a]=-\frac{i B}{\pi} \int d x a(\mathbf{x})$, and hence $j_{3, \epsilon}=\frac{1}{4 \pi^{2}} B$. To obtain the full response of the system, we need to add the (opposite) contribution of the second node and integrate over filled energy states up to some Fermi energy $\epsilon_{F}$. Taking into account that the existence of a bare linearization cutoff $\Lambda$ implies a cutoff $|\epsilon|<\left|\epsilon_{0} \pm \mu\right|, \epsilon_{0} \equiv v \Lambda$ for the accessible energy states (cf. Fig. 1), this leads to $j_{3}=\int_{-\epsilon_{0}+\mu}^{E_{F}} d \epsilon j_{3, \epsilon}-\int_{-\epsilon_{0}-\mu}^{\epsilon_{F}} d \epsilon j_{3, \epsilon}=\frac{\mu B}{2 \pi^{2}}$, i.e. an equilibrium current proportional to an external magnetic field, the so-called chiral magnetic effect (CME) [7, 8] (for a discussion how the nonvanishing of this expression may be understood from the perspective of the Fermi-liquid theory, see Ref. [20]).

Renormalization - What happens if short distance fluctuations in the field theory are integrated out to probe the physics at length scales beyond the ballistic/diffusive crossover regime? An answer to this question has been formulated in Ref. [17] within the framework of two loop renormalized perturbation theory for the dimensionless coupling constants $g_{\mu \nu}=\sigma_{\mu \nu} \Lambda^{-1}$ of the model. The result

$$
\frac{d g_{x x}}{d \ln l}=g_{x x}-\frac{1}{3 \pi^{4} g_{x x}}, \quad \frac{d g_{x y}}{d \ln l}=g_{x y},
$$

states that the longitudinal conductance scales according to the predictions of one-parameter scaling theory (unaffected by the Hall conductance) towards Ohmic behavior $g_{x x} \stackrel{g_{x x} \gg 1}{\sim} l$. The Hall conductance shows linear scaling, $g_{x y} \propto l$, which means that the AHE remains unrenormalized by disorder $\sigma_{x y}=$ const. even at large length scales (in contrast to the Hall conductivity of a two-dimensional system which is renormalized by instanton fluctuations [19]). Finally, the coupling constant of the CS action is fixed by gauge invariance, and fluctuation corrections to $\left\langle j_{3}\right\rangle_{B}$ vanish by supersymmetry. This means that within the framework of our theory the CME, too, fully protected against renormalization by disorder.

The disorder insensitivity of the topological response coeffecients holds regardless of whether we are probing the semimetallic Weyl nodes $\epsilon, \mu \sim \kappa$, or the metallic physics far away from them $|\epsilon \pm \mu| \gg \kappa$. The essential difference between the two situations lies in the bare and renormalized values of the longitudinal conductance $g_{x x}$ : in the former/latter case, $g_{x x}$ is initially small/large to begin with. However, in either case, $g_{x x}$ increases, and asymptotes to Ohmic behavior at large length scales. While the system then behaves similar to a three dimensional metal, the preserved non-vanishing of its two transverse transport coefficients betrays the underlying presence of two Dirac nodes.

Summarizing, we have microscopically derived a su- persymmetric field theory description of disordered Weyl semimetals and metals at length scales exceeding the mean free path. The profile of the theory is essentially determined by an interplay of symmetry conditions and the chiral anomaly. This mechanism stabilizes metallic behavior at large length scales, along with various disorderinsensitive response coefficients of topological origin.

Acknowledgments: We acknowledge discussions with $\mathrm{P}$. Brouwer, M. Hermanns, and S. Trebst. Work supported by SFB/TR 12 of the Deutsche Forschungsgemeinschaft. In the final stages of the preparation of this paper we became aware of arXiv:1412.7678 where an action similar to ours is motivated from a different perspective, viz. by dimensional reduction from a bulk $4 d$-topological insulator.

[1] P. Hosur and X. Qi, Comptes Rendus Physique 14, 857 (2013).

[2] B. Sbierski, G. Pohl, E. J. Bergholtz, and P. W. Brouwer, Phys. Rev. Lett. 113, 026602 (2014).

[3] A. A. Burkov, Phys. Rev. Lett. 113, 247203 (2014)

[4] S. V. Syzranov, L. Radzihovsky, and V. Gurarie, arXiv:1402.3737 (2014).

[5] S. V. Syzranov, L. Radzihovsky, and V. Gurarie, arXiv:1411.4635 (2014)

[6] A. Altland, B. Simons, and M. Zirnbauer, Physics Reports 359, 283 (2002)

[7] K. Fukushima, D. E. Kharzeev, and H. J. Warringa, Phys. Rev. D 78, 074033 (2008).

[8] Y. Chen, S. Wu, and A. A. Burkov, Phys. Rev. B 88, 125105 (2013)

[9] A. A. Burkov and L. Balents, Phys. Rev. Lett. 107, 127205 (2011)

[10] B. Roy and S. Das Sarma, Phys. Rev. B 90, 241112 (2014)

[11] E. Fradkin, Phys. Rev. B 33, 3263 (1986)

[12] K. B. Efetov, Sypersymmetry in Disorder and Chaos (Cambridge University Press, Cambridge, 1997).

[13] For $\gamma<\gamma^{*}$, the mean field equations have only a trivial solution $\kappa=0$, which is consistent with the presence of a critical point at $\sim \gamma^{*}$.

[14] A. N. Redlich, Phys. Rev. D 29, 2366 (1984).

[15] F. Wilczek and A. Zee, Phys. Rev. Lett. 51, 2250 (1983)

[16] G. E. Volovik and V. M. Yakovenko, Journal of Physics: Condensed Matter 1, 5263 (1989)

[17] Z. Wang, Phys. Rev. Lett. 79, 4002 (1997)

[18] Evidence that we start out on the metallic side of the transition follows from (i) the topological protection against localization, (ii) matching with the RG flow of the underlying $\psi$-field theory which is directed towards metallic behavior, and (iii) the fact that the RG analysis of the $\sigma$-model in $d=2+\epsilon, \epsilon=1$, dimensions predicts a value (cf. e.g. Ref.[17]) $g^{*}=1 / \sqrt{3} \pi^{2}$.

[19] H. Levine, S. B. Libby, and A. M. M. Pruisken, Nucl. Phys. B 240, 30 (1984)

[20] D. T. Son and N. Yamamoto, Phys. Rev. Lett. 109, $181602(2012)$ 